\title{
Confidence intervals centered on bootstrap smoothed estimators
}

\author{
Paul Kabaila* and Christeen Wijethunga \\ Department of Mathematics and Statistics, La Trobe University, Melbourne Victoria, \\ Australia.
}

\begin{abstract}
Bootstrap smoothed (bagged) parameter estimators have been proposed as an improvement on estimators found after preliminary data-based model selection. The key result of Efron (2014) is a very convenient and widely applicable formula for a delta method approximation to the standard deviation of the bootstrap smoothed estimator. This approximation provides an easily computed guide to the accuracy of this estimator. In addition, Efron (2014) proposed a confidence interval centered on the bootstrap smoothed estimator, with width proportional to the estimate of this approximation to the standard deviation. We evaluate this confidence interval in the scenario of two nested linear regression models, the full model and a simpler model, and a preliminary test of the null hypothesis that the simpler model is correct. We derive computationally convenient expressions for the ideal bootstrap smoothed estimator and the coverage probability and expected length of this confidence interval. In terms of coverage probability, this confidence interval outperforms the post-model-selection confidence interval with the same nominal coverage and based on the same preliminary test. We also compare the performance of confidence interval centered on the bootstrap smoothed estimator, in terms of expected length, to the usual confidence interval, with the same minimum coverage probablility, based on the full model.
\end{abstract}

Keywords: Bagging; Bootstrap smoothing; Coverage probability; Preliminary model selection.

* Corresponding author address: Department of Mathematics and Statistics, La Trobe University, Victoria 3086, Australia. Tel: +61-03-9479-2594; fax: +61-03-9479-2466. Email address: P.Kabaila@latrobe.edu.au 


\section{Introduction}

In applied statistics it is common practice to carry out preliminary data-based model selection (using e.g. hypothesis tests or minimizing a criterion such as AIC) and then to use the selected model to carry out further inference for the parameter of interest on the assumption that the selected model had been given to us a priori, as the true model. We refer to such further inferences as post-model-selection inferences. Postmodel-selection point estimators have the inherently undesirable property that they are discontinuous functions of the data. In the terminology of Efron (2014), they are "jumpy". Bootstrap smoothed (or bagged, Breiman, 1996) estimators have been proposed as an improvement on post-model-selection estimators. Bootstrap smoothed estimators are smoothed versions of the post-model-selection estimator. The key result of Efron (2014) is a new formula for a delta method approximation to the standard deviation of the bootstrap smoothed estimator. This formula is valid for any exponential family of models and has the attractive feature that it simply re-uses the parametric bootstrap replications that were employed to find this estimator. It also has the attractive feature that it is applicable in the context of complicated data-based model selection. This formula provides an easily computed guide to the accuracy of the bootstrap smoothed estimator.

Post-model-selection confidence intervals have the inherently undesirable property that they have endpoints that are discontinuous functions of the data. Furthermore, these confidence intervals may have minimum coverage probability far below nominal (see e.g. Leeb and Pötscher, 2005 and Kabaila, 2009). Confidence intervals that deal properly with the "model uncertainty" commonly encountered in applications are desperately needed by statistical practitioners. Such confidence intervals should have (a) endpoints that are smooth functions of the data, (b) have the desired minimum coverage probability and (c) attractive expected length properties.

In response to this need, a number of frequentist model averaged confidence intervals have been proposed (Buckland et al., 1997, Hjort and Claeskens, 2003, Fletcher and Turek, 2011, Turek and Fletcher, 2012). A related approach is the proposal of Efron (2014) of a confidence interval (CI) centered on the bootstrap smoothed estimator. This CI, with nominal coverage $1-\alpha$, has half-width equal to the $1-\alpha / 2$ quantile of the standard normal

distribution multiplied by the estimate of the delta method approximation, $\mathbf{s d}_{\text {delta }}$, to the 
standard deviation of this estimator. We call this interval the $\operatorname{sd}_{\text {delta }}$ interval.

Wang et al (2014) assess the $\mathrm{sd}_{\text {delta }}$ interval using simulations to estimate weighted averages over values of the explanatory variables of the coverage, center and length of this CI. In terms of these weighted averages, this CI seems to perform well for the scenarios that they consider. However, these weighted averages over the explanatory variables will tend to mask particular values of the explanatory variables for which the coverage is low or the expected length is large.

To rigorously evaluate the $s_{\text {delta }}$ interval, we consider the simple, though informative, scenario of two nested normal linear regression models and parameter of interest $\theta$ a specified linear combination of the regression parameters. These two nested models are the full model and the simpler model where $\tau$, a distinct specified linear combination of the regression parameters, is set to 0. This scenario was used by Kabaila, Welsh and Abeysekera (2016) and Kabaila, Welsh and Mainzer (2017) to evaluate the frequentist model averaged confidence intervals proposed by Fletcher and Turek (2011) and Turek and Fletcher (2012). The bootstrap smoothed estimator that we consider is a smoothed version of the post-model-selection estimator obtained after a preliminary test of the null hypothesis that $\tau=0$ against the alternative hypothesis that $\tau \neq 0$.

In Section 3, for this simple scenario of two nested regression models, we derive a computationally convenient exact expressions for the ideal (i.e. in the limit as the number of bootstrap simulations approaches infinity) bootstrap estimator. The delta-method approximation $\mathrm{sd}_{\text {delta }}$ to this standard deviation can be found using the formula of Efron (2014).

Let $\widehat{\theta}$ denote the least squares estimator of $\theta$ (based on the full model). The usual CI based on the full model is, of course, centered on $\widehat{\theta}$. Also let $\widehat{\tau}$ denote the least squares estimator of $\tau$ (based on the full model). In Section 4, we consider the coverage probability of the $s_{\text {delta }}$ interval. We show that this coverage probability is determined by the known correlation $\rho=\operatorname{corr}(\widehat{\theta}, \widehat{\tau})$ and the unknown parameter $\gamma=\tau /($ standard deviation of $\widehat{\tau})$. We also show that this coverage probability is an even function of $\gamma$, for every given $\rho$, and an even function of $\rho$, for every given $\gamma$. We are therefore able to encapsulate the coverage probability function of the $\mathrm{sd}_{\mathrm{delta}}$ interval, for all possible choices of design matrix, parameter of interest $\theta$ and parameter $\tau$ that specifies the simpler model, using only 
the two parameters $|\rho|$ and $|\gamma|$. An immediate consequence of the results of Section 3 is that when $\rho=0$, the $\operatorname{sd}_{\text {delta }}$ interval are identical to the usual CI, with actual coverage $1-\alpha$, based on the full model. However, as $|\rho|$ increases the latter confidence interval increasingly differs from the $\mathrm{sd}_{\text {delta }}$ interval.

Figure 1 shows the graph (solid line) of the coverage probability of the $\mathrm{sd}_{\text {delta }}$ interval centered on the bootstrap smoothed estimator based on the post-model-selection estimator obtained after a preliminary hypothesis test, with size 0.1 , of the null hypothesis that the simpler model is correct. This CI has nominal coverage 0.95. We consider $|\rho|=0.7$. Also shown in this figure is the graph (dashed line) of the coverage probability of the postmodel-selection CI with the same nominal coverage and based on the same preliminary test. This panel provides an illustration of the fact, established through an extensive numerical investigation described in the Supplementary material, that the $\operatorname{sd}_{\text {delta }}$ interval outperforms the post-model-selection CI, with the same nominal coverage and based on the same preliminary test, in terms of minimum coverage probability.

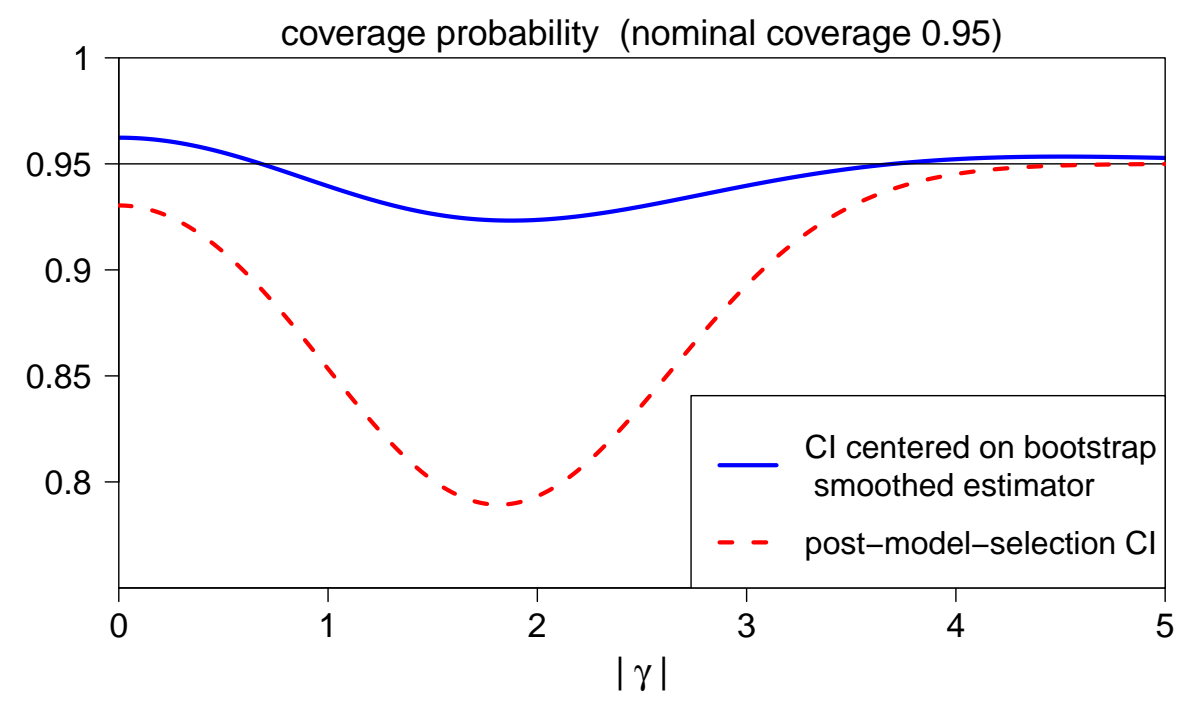

Figure 1: This figure shows a graph (dashed line) of the coverage probability of the post-model-selection CI, based on a preliminary hypothesis test, with size 0.1 , of the null hypothesis that the simpler model is correct. This CI has nominal coverage 0.95 . It also shows a graph (solid line) of the coverage probability for the sd $_{\text {delta }}$ interval, based on the post-model-selection estimator obtained after the same preliminary test. This CI also has nominal coverage 0.95 . Here $|\rho|=0.7$.

A further measure of the quality of the $s_{\text {delta }}$ interval is its scaled expected length, 
where the scaling is with respect to the expected length of the usual CI, with the same minimum coverage probability, based on the full model. In Section 5, we derive a computationally convenient formula for the scaled expected length of the $\mathrm{sd}_{\mathrm{delta}}$ interval. Using this formula, we provide a detailed examination of the scaled expected length properties of the sd $_{\text {delta }}$ interval.

\section{The two models and the post-model-selection estimator}

We consider two nested linear regression models: the full model $\mathcal{M}_{2}$ and the submodel $\mathcal{M}_{1}$. Suppose that the full model $\mathcal{M}_{2}$ is given by

$$
\boldsymbol{y}=\boldsymbol{X} \boldsymbol{\beta}+\varepsilon
$$

where $\boldsymbol{y}$ is a random $n$-vector of responses, $\boldsymbol{X}$ is a known $n \times p$ matrix with linearly independent columns $(p<n), \boldsymbol{\beta}$ is an unknown $p$-vector of parameters and $\boldsymbol{\varepsilon} \sim N\left(\mathbf{0}, \sigma^{2} \boldsymbol{I}_{n}\right)$ with $\sigma^{2}$ known. Suppose that $\boldsymbol{\beta}=\left[\theta, \tau, \boldsymbol{\lambda}^{\top}\right]^{\top}$, where $\theta$ is the scalar parameter of interest, $\tau$ is a scalar parameter used in specifying the model $\mathcal{M}_{1}$ and $\boldsymbol{\lambda}$ is a $(p-2)$-dimensional parameter vector. The model $\mathcal{M}_{1}$ is $\mathcal{M}_{2}$ with $\tau=0$. As shown in the Supplementary material, this scenario can be obtained by a change of parametrization from a more general scenario.

We assume that the error variance $\sigma^{2}$ is known, as does Efron (2014, Section 4) when he uses a linear regression model for the supernova data. It is highly plausible that for a linear regression model, the known $\sigma^{2}$ case provides a good approximation to the case that $\sigma^{2}$ is unknown, so that it must be estimated, and $n-p$ is reasonably large.

Let $\widehat{\boldsymbol{\beta}}$ denote the least squares estimator of $\boldsymbol{\beta}$, so that $\widehat{\boldsymbol{\beta}}=\left(\boldsymbol{X}^{\top} \boldsymbol{X}\right)^{-1} \boldsymbol{X}^{\top} \boldsymbol{y}$. Also let $\widehat{\theta}$ and $\widehat{\tau}$ denote the first and second components of $\widehat{\boldsymbol{\beta}}$, respectively. Let $v_{\theta}=\operatorname{var}(\widehat{\theta}) / \sigma^{2}$, $v_{\tau}=\operatorname{var}(\widehat{\tau}) / \sigma^{2}$ and $\rho=\operatorname{corr}(\widehat{\theta}, \widehat{\tau})$. Note that $v_{\theta}, v_{\tau}$ and $\rho$ are known. Let $\gamma=\tau /\left(\sigma v_{\tau}^{1 / 2}\right)$, which is an unknown parameter, and also let $\widehat{\gamma}=\widehat{\tau} /\left(\sigma v_{\tau}^{1 / 2}\right)$. We will express all quantities of interest in terms of the random vector $(\widehat{\theta}, \widehat{\gamma})$, which has a bivariate normal distribution with mean $(\theta, \gamma)$ and known covariance matrix.

Suppose that we carry out a preliminary test, of size $\widetilde{\alpha}$, of the null hypothesis $\tau=0$ against the alternative hypothesis $\tau \neq 0$. The test statistic is $|\widehat{\gamma}|$, which has the same distribution as $|Z|$, for $Z \sim N(0,1)$, under the null hypothesis. Let the quantile $z_{a}$ be defined by $P\left(Z \leq z_{a}\right)=a$ for $Z \sim N(0,1)$. We accept the null hypothesis when 
$|\widehat{\gamma}| \leq z_{1-\widetilde{\alpha} / 2}$; otherwise we reject the null hypothesis. In other words, if $|\widehat{\gamma}| \leq z_{1-\widetilde{\alpha} / 2}$ we choose model $\mathcal{M}_{1}$; otherwise we choose model $\mathcal{M}_{2}$.

The least squares estimators of $\theta$ under the models $\mathcal{M}_{2}$ and $\mathcal{M}_{1}$ are $\widehat{\theta}$ and $\widehat{\theta}-\rho \sigma v_{\theta}^{1 / 2} \widehat{\gamma}$, respectively. Therefore the post-model-selection estimator of $\theta$ is

$$
\widehat{\theta}_{\mathrm{PMS}}= \begin{cases}\widehat{\theta}-\rho \sigma v_{\theta}^{1 / 2} \widehat{\gamma} & \text { if }|\widehat{\gamma}| \leq z_{1-\widetilde{\alpha} / 2} \\ \widehat{\theta} & \text { otherwise. }\end{cases}
$$

For $\rho \neq 0$, we note that $\widehat{\theta}_{\text {PMS }}$ is (in the terminology of Efron, 2014) a "jumpy" estimate: as $|\widehat{\gamma}|$ increases through the value $z_{1-\widetilde{\alpha} / 2}, \widehat{\theta}_{\mathrm{PMS}}$ will change discontinuously. Henceforth, we suppose that the known quantities $\rho$ and $v_{\theta}$ and the size $\widetilde{\alpha}$ are given.

\section{Computationally convenient exact formulae for the ideal bootstrap smoothed estimate, standard deviation and delta-method approximation to the standard deviation}

Efron (2014) describes the ideal bootstrap smoothed estimate $\widetilde{\theta}$ of $\theta$ by considering a limit as the number of boostrap resamples $B \rightarrow \infty$. Because we are dealing with a parametric bootstrap, we are able to express the ideal bootstrap smoothed estimate as follows. Let $E_{\boldsymbol{\beta}}\left(\widehat{\theta}_{\mathrm{PMS}}\right)$ denote the expected value of $\widehat{\theta}_{\mathrm{PMS}}$, for true parameter value $\boldsymbol{\beta}$. The ideal bootstrap smoothed estimate $\tilde{\theta}$ is obtained by first evaluating $E_{\boldsymbol{\beta}}\left(\widehat{\theta}_{\mathrm{PMS}}\right)$ and then replacing $\boldsymbol{\beta}$ by $\widehat{\boldsymbol{\beta}}$.

The following theorem, proved in the appendix, provides a computationally convenient exact formula for $E_{\boldsymbol{\beta}}\left(\widehat{\theta}_{\mathrm{PMS}}\right)$. Let $\Phi$ and $\phi$ denote the $N(0,1)$ cumulative distribution function and probability density function, respectively.

Theorem 1. Let $k(\gamma)=\phi(d+\gamma)-\phi(d-\gamma)+\gamma[\Phi(d-\gamma)-\Phi(-d-\gamma)]$. Then $E_{\boldsymbol{\beta}}\left(\widehat{\theta}_{\mathrm{PMS}}\right)=$ $\theta-\rho \sigma v_{\theta}^{1 / 2} k(\gamma)$. Note that $k(0)=0$ and $k(\gamma)$ is an odd function of $\gamma$ that takes positive values for all $\gamma>0$ and approaches 0 as $\gamma \rightarrow \infty$.

It follows from this theorem that the ideal bootstrap smoothed estimator $\widetilde{\theta}$ satisfies

$$
\widetilde{\theta}=\widehat{\theta}-\rho \sigma v_{\theta}^{1 / 2} k(\widehat{\gamma})
$$

The following theorem, proved in the appendix, provides a computationally convenient

exact formula for the standard deviation of $\widetilde{\theta}$. We denote this standard deviation by $\operatorname{sd}(\gamma)$. 
Theorem 2. The standard deviation of $\widetilde{\theta}$ is a function of $\gamma$, which we denote by $\operatorname{sd}(\gamma)$, is $\sigma v_{\theta}^{1 / 2} r(\gamma ; \rho)$, where

$r(\gamma ; \rho)=\left(1-2 \rho^{2} \int_{-\infty}^{\infty} k(z)(z-\gamma) \phi(z-\gamma) d z+\rho^{2} \int_{-\infty}^{\infty}\left(k(z)-m_{k}(\gamma)\right)^{2} \phi(z-\gamma) d z\right)^{1 / 2}$ for

$$
m_{k}(\gamma)=\int_{-\infty}^{\infty} k(z) \phi(z-\gamma) d z
$$

The following theorem, proved in the appendix, provides a computationally convenient exact formula for the delta-method approximation to the standard deviation of the ideal bootstrap smoothed estimator $\widetilde{\theta}$.

Theorem 3. Let $q(\gamma)=\Phi(d-\gamma)-\Phi(-d-\gamma)-d[\phi(d+\gamma)+\phi(d-\gamma)]$. Note that $q(\gamma)$ is an even function of $\gamma$. The delta-method approximation to the standard deviation of $\tilde{\theta}$ is a function of $\gamma$, which we denote by $\operatorname{sd}_{\text {delta }}(\gamma)$, and is $\sigma v_{\theta}^{1 / 2} r_{\text {delta }}(\gamma ; \rho)$, where

$$
r_{\text {delta }}(\gamma ; \rho)=\left(1-2 \rho^{2} q(\gamma)+\rho^{2} q^{2}(\gamma)\right)^{1 / 2}
$$

We consider the following confidence intervals for $\theta$ centered on the bootstrap smoothed esimator $\widetilde{\theta}$, with nominal coverage $1-\alpha$ :

$$
\begin{aligned}
J & =\left[\widetilde{\theta}-z_{1-\alpha / 2} \operatorname{sd}(\widehat{\gamma}), \widetilde{\theta}+z_{1-\alpha / 2} \operatorname{sd}(\widehat{\gamma})\right] & (\text { sd interval }) \\
J_{\text {delta }} & =\left[\widetilde{\theta}-z_{1-\alpha / 2} \operatorname{sd}_{\text {delta }}(\widehat{\gamma}), \widetilde{\theta}+z_{1-\alpha / 2} \operatorname{sd}_{\text {delta }}(\widehat{\gamma})\right] & \left(\operatorname{sd}_{\text {delta }} \text { interval }\right)
\end{aligned}
$$

\section{Coverage probability of the confidence interval centered on the bootstrap smoothed estimator}

Let $C P(\gamma, \rho)$ and $C P_{\text {delta }}(\gamma, \rho)$ denote the coverage probabilities $P(\theta \in J)$ and $P(\theta \in$ $\left.J_{\text {delta }}\right)$, respectively. Also let $\Phi(\ell, u ; \mu, v)=P(\ell \leq Z \leq u)$ for $Z \sim N(\mu, v)$. The following theorem is proved in the appendix.

Theorem 4. Let $\ell(\gamma, \rho)=-z_{1-\alpha / 2} r(\gamma ; \rho)+\rho k(\gamma)$ and $u(\gamma, \rho)=z_{1-\alpha / 2} r(\gamma ; \rho)+\rho k(\gamma)$. Then

(a)

$$
C P(\gamma, \rho)=\int_{-\infty}^{\infty} \Phi\left(\ell(h, \rho), u(h, \rho) ; \rho(h-\gamma), 1-\rho^{2}\right) \phi(h-\gamma) d h,
$$

(b) For every given $\rho, C P(\gamma, \rho)$ is an even function of $\gamma$ and, for every given $\gamma, C P(\gamma, \rho)$ is an even function of $\rho$. 
The proof of the following theorem is the same as the proof of Theorem 4 , but with $r(\gamma ; \rho)$ replaced by $r_{\text {delta }}(\gamma ; \rho)$.

Theorem 5. Let $\ell_{\text {delta }}(\gamma, \rho)=-z_{1-\alpha / 2} r_{\text {delta }}(\gamma ; \rho)+\rho k(\gamma)$ and $u_{\text {delta }}(\gamma, \rho)=z_{1-\alpha / 2} r_{\text {delta }}(\gamma ; \rho)+\rho k(\gamma)$. Then

(a)

$$
C P_{\text {delta }}(\gamma, \rho)=\int_{-\infty}^{\infty} \Phi\left(\ell_{\text {delta }}(h, \rho), u_{\text {delta }}(h, \rho) ; \rho(h-\gamma), 1-\rho^{2}\right) \phi(h-\gamma) d h .
$$

(b) For every given $\rho, C P_{\text {delta }}(\gamma, \rho)$ is an even function of $\gamma$ and, for every given $\gamma$, $C P_{\text {delta }}(\gamma, \rho)$ is an even function of $\rho$.

\section{Scaled expected length of the confidence interval centered on the bootstrap smoothed estimator}

The scaled expected length of the confidence interval $J$, with nominal coverage $1-\alpha$, is defined as follows. Let $c_{\min }$ denote the minimum coverage probability of this confidence interval. Now let $I(c)$ denote the usual confidence interval for $\theta$, with coverage $c$, based on the full model. In other words, let $I(c)=\left[\widehat{\theta}-z_{(1+c) / 2} \sigma v_{\theta}^{1 / 2}, \widehat{\theta}+\right.$ $\left.z_{(1+c) / 2} \sigma v_{\theta}^{1 / 2}\right]$. The scaled expected length of $J$, denoted $S E L(\gamma, \rho)$, is defined to be the ratio $E$ (length of $J) / E$ (length of $\left.I\left(c_{\min }\right)\right)$. The following theorem is proved in the appendix.

Theorem 6. Let $c_{\min }$ denote the minimum coverage probability of the confidence interval $J$, with nominal coverage $1-\alpha$. Then

(a)

$$
\operatorname{SEL}(\gamma, \rho)=\frac{z_{1-\alpha / 2}}{z_{\left(1+c_{\min }\right) / 2}} \int_{-\infty}^{\infty} r(h ; \rho) \phi(h-\gamma) d h .
$$

(b) For every given $\rho, S E L(\gamma, \rho)$ is an even function of $\gamma$ and, for every given $\gamma, S E L(\gamma, \rho)$ is an even function of $\rho$.

The scaled expected length of the confidence interval $J_{\text {delta }}$, denoted by $S E L_{\text {delta }}(\gamma, \rho)$, is defined in a similar way to the scaled expected length of $J$. The proof of the following theorem is the same as the proof of Theorem 6 , but with $r(\gamma ; \rho)$ replaced by $r_{\text {delta }}(\gamma ; \rho)$.

Theorem 7. Let $c_{\min }$ denote the minimum coverage probability of the confidence interval $J_{\text {delta, }}$ with nominal coverage $1-\alpha$. Then 
(a)

$$
S E L_{\text {delta }}(\gamma, \rho)=\frac{z_{1-\alpha / 2}}{z_{\left(1+c_{\min }\right) / 2}} \int_{-\infty}^{\infty} r_{\text {delta }}(h ; \rho) \phi(h-\gamma) d h
$$

(b) For every given $\rho, S E L_{\mathrm{delta}}(\gamma, \rho)$ is an even function of $\gamma$ and, for every given $\gamma$, $S E L_{\text {delta }}(\gamma, \rho)$ is an even function of $\rho$.

It follows from Theorems 6 and 7 that we are able to encapsulate the scaled expected length of both the sd interval and the $s_{\text {delta }}$ interval, for all possible choices of design matrix, parameter of interest $\theta$ and parameter $\tau$ that specifies the simpler model, using only the two parameters $|\rho|$ and $|\gamma|$.

The bootstrap smoothed estimator is obtained by smoothing the post-model-selection estimator that results from a preliminary test of the null hypothesis that the simpler model is correct i.e. that $\gamma=0$. This post-model-selection estimator is usually motivated by a desire for good performance when the simpler model is correct. Therefore, ideally, both the sd interval and the $s_{\text {delta }}$ interval should have a scaled expected length that is substantially less than 1 when $\gamma=0$. In addition, ideally, these confidence intervals should have scaled expected length that (a) has maximum value that is not too much larger than 1 and (b) approaches 1 as $|\gamma|$ approaches infinity.

Figure 2 is the graph of the scaled expected length of the CI centred on the bootstrap smoothed estimator, which is based on the post-model-selection estimator obtained after a preliminary hypothesis test, with size 0.1 , of the null hypothesis that the simpler model is correct. This CI has nominal coverage 0.95 and width proportional to the estimate of $\operatorname{sd}_{\text {delta }}$ (obtained by replacing $\gamma$ by $\widehat{\gamma}$ in the expression for $\operatorname{sd}_{\text {delta }}$ ). We consider $|\rho|=$ 0.2, 0.5, 0.7 and 0.9. This figure provides an illustration of the following two properties of CI's centred on the bootstrap smoothed estimator, with width proportional to the estimate of either sd or $\operatorname{sd}_{\text {delta }}$ (obtained by replacing $\gamma$ by $\widehat{\gamma}$ ). The scaled expected lengths of these CI's (a) are either greater than 1 or only slightly less than 1 at $\gamma=0$ and (b) have maximum values that are increasing functions of $|\rho|$ that can be much larger than 1 for $|\rho|$ large. These properties are established, through extensive numerical evaluation, in the Supplementary material. Our overall interpretation of these two properties is that the CI centred on the bootstrap smoothed estimator, with width proportional to the estimated standard deviation, does not perform substantially better than the the usual confidence 
interval, with the same minimum coverage probability, based on the full model.

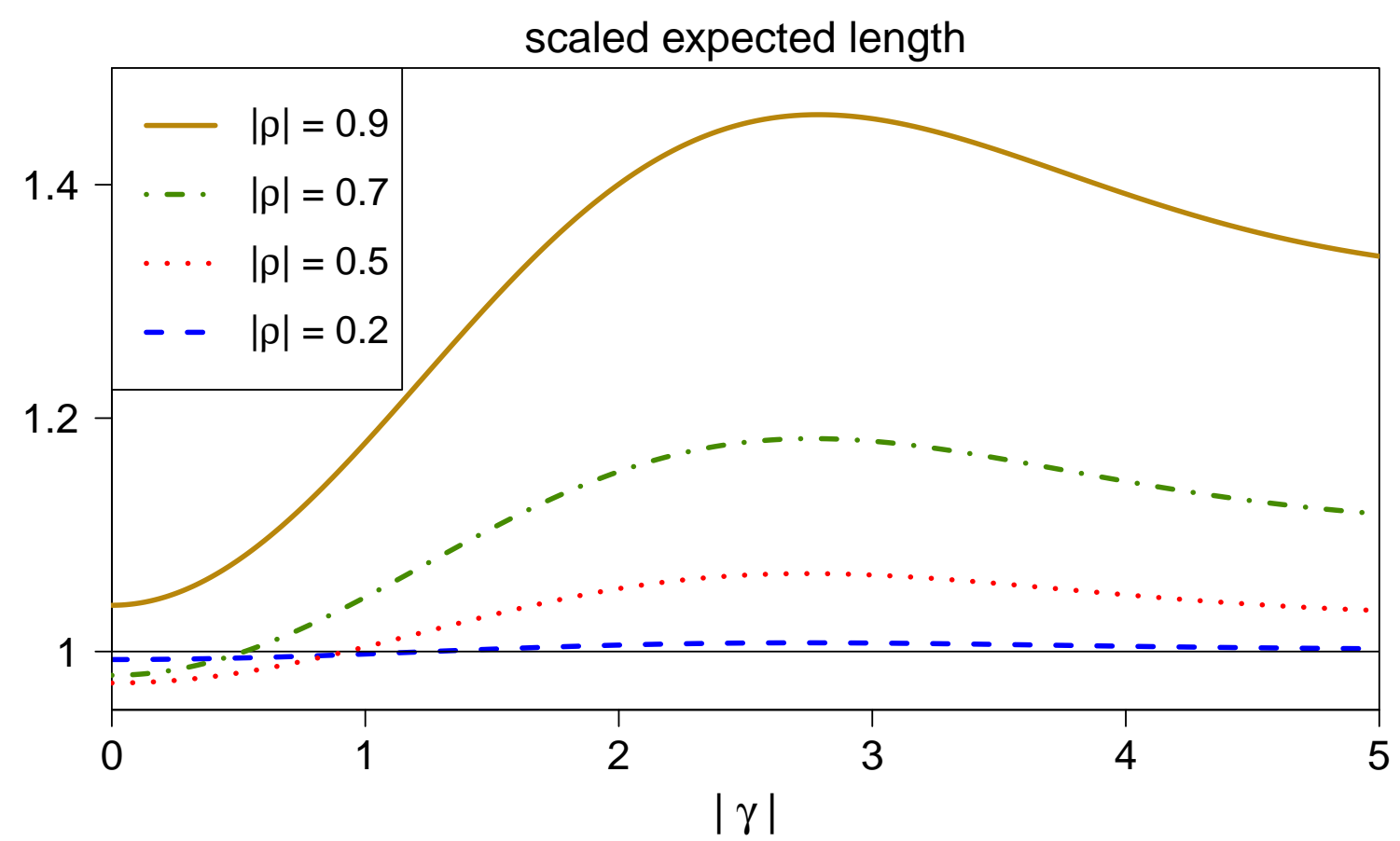

Figure 2: Graphs, for $|\rho|=0.2,0.5,0.7$ and 0.9 , of the scaled expected length of the sd $_{\text {delta }}$ interval, which is based on the post-model-selection estimator obtained after a preliminary hypothesis test, with size 0.1 , of the null hypothesis that the simpler model is correct. This CI has nominal coverage 0.95 .

\section{Discussion}

We have considered the scenario of two nested linear regression models, with the model chosen using a preliminary test. We have considered the case of known error variance, which is an approximation to the case that the error variance is unknown and the usual estimator of this variance is reasonably accurate. Also, under the appropriate large sample conditions, a logistic regression can be transformed, to a good approximation, to a linear regression model with normal errors having known error variance (see e.g. Cox, 1970, Chapter 3).

The advantage of the scenario that we consider is that we have derived computationally convenient exact expressions for all of the quantities of interest. This, in turn, has allowed us to make findings in this scenario that are valid for all design matrices, all parameters 
of interest that are linear combinations of the regression parameter vector, all possible preliminary tests with all possible test levels.

Usually, in practice, the bootstrap smoothed estimator is found by using a finite number $B$ of bootstrap resamples. This estimator is a "noisy" version of the ideal bootstrap smoothed estimator, which is found in the limit as $B \rightarrow \infty$. We consider the ideal bootstrap smoothed estimator and so we have placed the bootstrap smoothed estimator in the best possible light.

We have considered a confidence interval, with nominal coverage $1-\alpha$ and with half-width equal to the $1-\alpha / 2$ quantile of the standard normal distribution multiplied by the estimate of the standard deviation of this estimator. We call this the sd interval. We have also considered the same confidence interval, but with this standard deviation replaced by the delta method approximation to it. We call this interval the $\mathrm{sd}_{\text {delta }}$ interval. We have shown that both of these confidence intervals outperform the post-model-selection confidence interval, with the same nominal coverage and based on the same preliminary test, in terms of minimum coverage probability.

We have found, however, that the sdinterval and $s_{\text {delta }}$ interval do not perform any better in terms of expected length than the usual confidence interval, with the same minimum coverage probability and based on the full model. This is consistent with the observation by Hjort (2014) that one would expect to be able to improve on the $\mathrm{sd}_{\mathrm{delta}}$ interval because the distribution of the difference between the bootstrap smoothed estimator and the true parameter value is "typically highly nonnormal, asymmetric etc." The choice of data-based width of a confidence interval centred on the bootstrap smoothed estimator has a crucial role in determining the performance, in terms

of the coverage and expected length, of this interval. Our conclusion is that finding a good recipe for this data-based width is still an open problem.

\section{Appendix: Proofs of Theorems 1, 2, 3, 4 and 6}

In this appendix we prove Theorems 1, 2, 3, 4 and 6 . These proofs use the following lemma.

\section{Lemma 1.}

$$
\left[\begin{array}{l}
\widehat{\theta} \\
\widehat{\gamma}
\end{array}\right] \sim N\left(\left[\begin{array}{l}
\theta \\
\gamma
\end{array}\right],\left[\begin{array}{cc}
\sigma^{2} v_{\theta} & \rho \sigma v_{\theta}^{1 / 2} \\
\rho \sigma v_{\theta}^{1 / 2} & 1
\end{array}\right]\right)
$$




\section{Proof of Theorem 1}

To make the dependence of $\widehat{\theta}_{\mathrm{PMS}}$ on $\widehat{\theta}$ and $\widehat{\gamma}$ explicit, we write $\widehat{\theta}_{\mathrm{PMS}}=h(\widehat{\theta}, \widehat{\gamma})$. Now

$$
\begin{aligned}
E_{\boldsymbol{\beta}}\left(\widehat{\theta}_{\mathrm{PMS}}\right)= & \int_{-\infty}^{\infty} E_{\boldsymbol{\beta}}(h(\widehat{\theta}, z) \mid \widehat{\gamma}=z) \phi(z-\gamma) d z, \quad \text { since } \widehat{\gamma} \sim N(\gamma, 1), \\
= & \int_{-d}^{d} E_{\boldsymbol{\beta}}(h(\widehat{\theta}, z) \mid \widehat{\gamma}=z) \phi(z-\gamma) d z+\int_{-\infty}^{-d} E_{\boldsymbol{\beta}}(h(\widehat{\theta}, z) \mid \widehat{\gamma}=z) \phi(z-\gamma) d z \\
& \quad+\int_{d}^{\infty} E_{\boldsymbol{\beta}}(h(\widehat{\theta}, z) \mid \widehat{\gamma}=z) \phi(z-\gamma) d z \\
= & \int_{-d}^{d} E_{\boldsymbol{\beta}}\left(\widehat{\theta}-\rho \sigma v_{\theta}^{1 / 2} z \mid \widehat{\gamma}=z\right) \phi(z-\gamma) d z \\
& \quad+\int_{-\infty}^{-d} E_{\boldsymbol{\beta}}(\widehat{\theta} \mid \widehat{\gamma}=z) \phi(z-\gamma) d z+\int_{d}^{\infty} E_{\boldsymbol{\beta}}(\widehat{\theta} \mid \widehat{\gamma}=z) \phi(z-\gamma) d z, \text { by (2), } \\
= & \int_{-\infty}^{\infty} E_{\boldsymbol{\beta}}(\widehat{\theta} \mid \widehat{\gamma}=z) \phi(z-\gamma) d z-\rho \sigma v_{\theta}^{1 / 2} \int_{-d}^{d} z \phi(z-\gamma) d z \\
= & E_{\boldsymbol{\beta}}(\widehat{\theta})-\rho \sigma v_{\theta}^{1 / 2} \int_{-d}^{d} z \phi(z-\gamma) d z \\
= & \theta-\rho \sigma v_{\theta}^{1 / 2} k(\gamma),
\end{aligned}
$$

where

$$
k(\gamma)=\int_{-d}^{d} z \phi(z-\gamma) d z .
$$

The formula for and properties of $k(\gamma)$ stated in the theorem are proved in the Supplementary material.

\section{Proof of Theorem 2}

It follows from $(3)$ that

$$
\begin{aligned}
\operatorname{var}(\widetilde{\theta}) & =\operatorname{var}(\widehat{\theta})+\rho^{2} \sigma^{2} v_{\theta} \operatorname{var}(k(\widehat{\gamma}))-2 \rho \sigma v_{\theta}^{1 / 2} E((\widehat{\theta}-\theta)(k(\widehat{\gamma})-E(k(\widehat{\gamma})))) \\
& =\sigma^{2} v_{\theta}+\rho^{2} \sigma^{2} v_{\theta} \int_{-\infty}^{\infty}\left(k(z)-m_{k}(\gamma)\right)^{2} \phi(z-\gamma) d z-2 \rho \sigma^{2} v_{\theta} E(G k(\widehat{\gamma})),
\end{aligned}
$$

where $G=(\widehat{\theta}-\theta) /\left(\sigma v_{\theta}^{1 / 2}\right)$. Now $E(G k(\widehat{\gamma}) \mid \widehat{\gamma}=z)=k(z) E(G \mid \widehat{\gamma}=z)=\rho k(z)(z-\gamma)$, since

$$
\left[\begin{array}{l}
G \\
\widehat{\gamma}
\end{array}\right] \sim N\left(\left[\begin{array}{l}
0 \\
\gamma
\end{array}\right],\left[\begin{array}{ll}
1 & \rho \\
\rho & 1
\end{array}\right]\right)
$$

by Lemma 1 . Thus

$$
E(G k(\widehat{\gamma}))=\rho \int_{-\infty}^{\infty} k(z)(z-\gamma) \phi(z-\gamma) d z
$$




\section{Proof of Theorem 3}

To prove that $q$ is an even function, we need to prove that

$$
q(-\gamma)=\Phi(d+\gamma)-\Phi(-d+\gamma)-d[\phi(-d+\gamma)+\phi(d+\gamma)]
$$

is equal to

$$
q(\gamma)=\Phi(d-\gamma)-\Phi(-d-\gamma)-d[\phi(-d-\gamma)+\phi(d-\gamma)]
$$

Since $\Phi(z)=1-\Phi(-z), \Phi(d-\gamma)-\Phi(-d-\gamma)=\Phi(d+\gamma)-\Phi(-d+\gamma)$. The result follows from $\phi(-d+\gamma)+\phi(d+\gamma)=\phi(-d-\gamma)+\phi(d-\gamma)$, since $\phi$ is an even function.

The formula for $\operatorname{sd}_{\text {delta }}(\gamma)$ can be derived using Theorem 2 of Efron (2014). However, in the present scenario, the same formula results from the application of the delta-method approximation that uses the first order Taylor expansion, $k(\widehat{\gamma}) \approx k(\gamma)+k^{\prime}(\gamma)(\widehat{\gamma}-\gamma)$. It follows from (3) that

$$
\widetilde{\theta} \approx \widehat{\theta}-\rho \sigma v_{\theta}^{1 / 2}\left(k(\gamma)+k^{\prime}(\gamma)(\widehat{\gamma}-\gamma)\right)
$$

The variance of the right-hand side is $\sigma^{2} v_{\theta}\left(1-2 \rho^{2} k^{\prime}(\gamma)+\rho^{2}\left(k^{\prime}(\gamma)\right)^{2}\right)$. Using the definition of Hermite polynomials, it may be shown that $k^{\prime}(\gamma)=q(\gamma)$.

\section{Proof of Theorem 4}

\section{Part (a)}

$$
\begin{aligned}
P(\theta \in J) & =P\left(-z_{1-\alpha / 2} \operatorname{sd}(\widehat{\gamma}) \leq \widetilde{\theta}-\theta \leq z_{1-\alpha / 2} \operatorname{sd}(\widehat{\gamma})\right) \\
& =P\left(-z_{1-\alpha / 2} \operatorname{sd}(\widehat{\gamma}) \leq \widehat{\theta}-\theta-\rho \sigma v_{\theta}{ }^{1 / 2} k(\widehat{\gamma}) \leq z_{1-\alpha / 2} \operatorname{sd}(\widehat{\gamma})\right), \text { by (3)), } \\
& =P\left(-z_{1-\alpha / 2} \frac{\operatorname{sd}(\widehat{\gamma})}{\sigma v_{\theta}^{1 / 2}} \leq G-\rho k(\widehat{\gamma}) \leq z_{1-\alpha / 2} \frac{\operatorname{sd}(\widehat{\gamma})}{\sigma v_{\theta}^{1 / 2}}\right), \text { where } G=(\widehat{\theta}-\theta) /\left(\sigma v_{\theta}{ }^{1 / 2}\right) \\
& =P(\ell(\widehat{\gamma}, \rho) \leq G \leq u(\widehat{\gamma}, \rho)) \\
& =\int_{-\infty}^{\infty} P(\ell(h, \rho) \leq G \leq u(h, \rho) \mid \widehat{\gamma}=h) \phi(h-\gamma) d h .
\end{aligned}
$$

It follows from (5) that the distribution of $G$ conditional on $\widehat{\gamma}=h$ is $N\left(\rho(h-\gamma), 1-\rho^{2}\right)$. Hence

$$
\int_{-\infty}^{\infty} P(\ell(h, \rho) \leq G \leq u(h, \rho) \mid \widehat{\gamma}=h) \phi(h-\gamma) d h=\int_{-\infty}^{\infty} P(\ell(h, \rho) \leq \widetilde{G} \leq u(h, \rho)) \phi(h-\gamma) d h
$$

where $\widetilde{G} \sim N\left(\rho(h-\gamma), 1-\rho^{2}\right)$. Therefore (4) holds.

Part (b): Our proof will use the following easily-established lemmas. 
Lemma 2. $\Phi(\ell, u ; \mu, v)=\Phi(-u,-\ell ;-\mu, v)$.

Lemma 3. (a) $-u(-x, \rho)=\ell(x, \rho)$.

(b) $\ell(x,-\rho)=\ell(-x, \rho)$ and $u(x,-\rho)=u(-x, \rho)$.

Firstly, we prove that, for every given $\rho, C P(\gamma, \rho)$ is an even function of $\gamma$. By Lemma 2 and since $\phi$ is an even function,

$$
\begin{aligned}
C P(-\gamma, \rho) & =\int_{-\infty}^{\infty} \Phi\left(-u(h, \rho),-\ell(h, \rho) ; \rho(-h-\gamma), 1-\rho^{2}\right) \phi(-h-\gamma) d h \\
& =\int_{-\infty}^{\infty} \Phi\left(-u(-x, \rho),-\ell(-x, \rho) ; \rho(x-\gamma), 1-\rho^{2}\right) \phi(x-\gamma) d x \\
& =\int_{-\infty}^{\infty} \Phi\left(\ell(x, \rho), u(x, \rho) ; \rho(x-\gamma), 1-\rho^{2}\right) \phi(x-\gamma) d x, \text { by Lemma } 3 \text { (a), } \\
& =C P(\gamma, \rho)
\end{aligned}
$$

We now prove that, for every given $\gamma, C P(\gamma, \rho)$ is an even function of $\rho$. Now

$$
\begin{aligned}
C P(\gamma,-\rho) & =\int_{-\infty}^{\infty} \Phi\left(\ell(h,-\rho), u(h,-\rho) ;-\rho(h-\gamma), 1-\rho^{2}\right) \phi(h-\gamma) d h \\
& =\int_{-\infty}^{\infty} \Phi\left(-u(h,-\rho),-\ell(h,-\rho) ; \rho(h-\gamma), 1-\rho^{2}\right) \phi(h-\gamma) d h, \text { by Lemma } 2, \\
& =\int_{-\infty}^{\infty} \Phi\left(\ell(h, \rho), u(h, \rho) ; \rho(h-\gamma), 1-\rho^{2}\right) \phi(h-\gamma) d h, \text { by Lemma } 3, \\
& =C P(\gamma, \rho)
\end{aligned}
$$

\section{Proof of Theorem 6}

Part (a): By Theorem 2, the length of the confidence interval $J$, with nominal coverage $1-\alpha$, is $2 z_{1-\alpha / 2} \sigma v_{\theta}^{1 / 2} r(\widehat{\gamma} ; \rho)$. Thus the expected length of this CI is $2 z_{1-\alpha / 2} \sigma v_{\theta}^{1 / 2} E(r(\widehat{\gamma}, \rho))$. Also, the length of $I\left(c_{\min }\right)$ is $2 z_{\left(1+c_{\min }\right) / 2} \sigma v_{\theta}^{1 / 2}$. Thus

$$
S E L(\gamma, \rho)=\frac{z_{1-\alpha / 2}}{z_{\left(1+c_{\min }\right) / 2}} E(r(\widehat{\gamma}, \rho))=\frac{z_{1-\alpha / 2}}{z_{\left(1+c_{\min }\right) / 2}} \int_{-\infty}^{\infty} r(h ; \rho) \phi(h-\gamma) d h .
$$

Part (b): Our proof will use the following lemma.

Lemma 4. For every given $\rho, r(\gamma ; \rho)$ is an even function of $\gamma$ and, for every given $\gamma$, $r(\gamma ; \rho)$ is an even function of $\rho$. 
Since $\phi$ is an even function,

$$
\begin{aligned}
S E L(-\gamma, \rho) & =\frac{z_{1-\alpha / 2}}{z_{\left(1+c_{\min }\right) / 2}} \int_{-\infty}^{\infty} r(h ; \rho) \phi(-h-\gamma) d h \\
& =\frac{z_{1-\alpha / 2}}{z_{\left(1+c_{\min }\right) / 2}} \int_{-\infty}^{\infty} r(-x ; \rho) \phi(x-\gamma) d x \\
\quad(\text { by changing the variable of integration to } x=-h) & \\
& =S E L(\gamma, \rho),
\end{aligned}
$$

by Lemma 4. It also follows directly from this lemma that $\operatorname{SEL}(\gamma, \rho)$ is an even function of $\rho$, for every given $\gamma$.

\section{References}

Breiman, L. (1996). Bagging predictors. Machine Learning 24, 123-140

Buckland, S.T., Burnham, K.P. and Augustin, N.H. (1997). Model selection: an integral part of inference. Biometrics 53, 603-618.

Cox, D.R. (1970). The Analysis of Binary Data. Methuen, London.

Efron, B. (2014). Estimation and accuracy after model selection. Journal of the American Statistical Association 109, 991-1022.

Fletcher, D., Turek, D. (2011). Model-averaged profile likelihood intervals. Journal of Agricultural, Biological and Environmental Statistics 17, 38-51.

Hjort, N.L. (2014). Comment on 'Estimation and accuracy after model selection' by B. Efron. Journal of the American Statistical Association 109, 1017-1020.

Hjort, N.L. and Claeskens, G. (2003). Frequentist model average estimators. Journal of the American Statistical Association 98, 879-899.

Kabaila, P. (2009). The coverage properties of confidence regions after model selection. International Statistical Review 77, 405-414.

Kabaila, P. (2016). The finite sample performance of the two-stage analysis of a twoperiod crossover trial. Statistics and Probability Letters, 117, 118-127.

Kabaila, P., Welsh, A.H. and Abeysekera, W. (2016). Model-averaged confidence intervals. Scandinavian Journal of Statistics 43, 35-48. 
Kabaila, P., Welsh, A.H. and Mainzer, R. (2016). The performance of model averaged tail area confidence intervals. Communications in Statistics - Theory and Methods, 46, 10718-10732.

Leeb, H. and Pötscher, B.M. (2005). Model selection and inference: facts and fiction. Econometric Theory 21, 21-59.

Turek, D. and Fletcher, D. (2012). Model-averaged Wald confidence intervals. Computational Statistics and Data Analysis 56, 2809-2815.

Wang, L., Sherwood, B. and Li, R. (2014). Comment on 'Estimation and accuracy after model selection' by B. Efron. Journal of the American Statistical Association 109, $1007-1010$. 